\section{Acesso e utilização dos serviços de saúde bucal por idosos de Pelotas, Rio Grande do Sul, Brasil}

\author{
Access to and use of oral health services among \\ the elderly in Pelotas, Rio Grande do Sul State, \\ Brazil
}

Acceso y utilización de los servicios de salud bucal por parte de ancianos en Pelotas, Río Grande do Sul, Brasil

\section{Resumo}

O objetivo foi medir a falta de acesso e a utilização dos serviços de saúde bucal por idosos de Pelotas, Rio Grande do Sul, Brasil. Estudo transversal de base populacional foi realizado no ano de 2014, na zona urbana do município, incluindo indivíduos com 60 anos e mais. Variáveis sociodemográficas e de necessidade autorreferidas foram associadas aos desfechos. Utilizou-se regressão de Poisson para as análises bruta e ajustada. Foram entrevistados 1.451 idosos mediante um questionário estruturado. Falta de acesso no último ano alcançou uma prevalência de 1,8\% (IC95\%: 0,7-3,0). Idosos que nunca consultaram somaram 3,1\% (IC95\%: 2,2-4,0) e a utilização de serviços de saúde bucal no último ano registrou prevalência de 38,3\% (IC95\%: 36,0-41,0). A utilização no último ano apresentou associação positiva com as seguintes variáveis: faixa etária mais jovem $(R P=1,16)$, ter companheiro $(R P=1,28)$, alta escolaridade $(R P=1,31)$, problema na boca ou nos dentes $(R P=1,93)$, necessidade de prótese dentária $(R P=1,36)$ e ser edêntulo $(R P=3,11)$. A falta de acesso no último ano foi baixa. A utilização de serviços de saúde bucal foi mais alta do que a observada em outros estudos. Os achados parecem refletir a expansão desses serviços, particularmente na rede pública, sendo úteis para as ações de planejamento das políticas de saúde.

Acesso aos Serviços de Saúde; Equidade no Acesso aos Serviços de Saúde; Serviços de Saúde para Idosos; Serviços de Saúde Bucal
Giordano Santana Sória 1

Bruno Pereira Nunes 1

Caren Serra Bavaresco 2

Luna Strieder Vieira 1

Luiz Augusto Facchini 1

doi: 10.1590/0102-311X00191718

\section{Correspondência}

L.S. Vieira

Universidade Federal de Pelotas.

Rua Marechal Deodoro 1160, 3o piso, Pelotas, RS 96020-220, Brasil.

luna.stri@gmail.com

1 Universidade Federal de Pelotas, Pelotas, Brasil.

2 Universidade Luterana do Brasil, Canoas, Brasil. 


\section{Introdução}

O acesso aos serviços é um dos principais objetivos dos sistemas de saúde, sendo alvo de amplo debate por gestores e acadêmicos após a reforma sanitária brasileira. Essa discussão ficou mais intensa após as mudanças políticas e sociais realizadas no Brasil que culminaram com a criação do Sistema Único de Saúde (SUS), que garante a universalidade como um direito do cidadão ${ }^{1}$. Entretanto, a operacionalização do acesso em pesquisas populacionais ainda é empregada de forma imprecisa e variada, confundindo-se com o conceito de utilização dos serviços de saúde 2,3.

Existe uma interseção entre os conceitos de acesso e utilização, sendo o primeiro relativo ao direito pleno de ingresso no sistema de saúde sem obstáculos físicos, financeiros ou culturais, decorrentes de uma necessidade percebida 2,3,4. Já a utilização dos serviços de saúde representa o acesso efetivado, a obtenção da busca por atendimento, resultando do comportamento do indivíduo que procura cuidados e de características do serviço, tanto organizacionais, quanto dos profissionais, que conduzem o usuário pelo sistema. Ainda que a utilização seja considerada por alguns autores um proxy de acesso, informações sobre os indivíduos que não conseguiram acessar os serviços são escassas; possivelmente a principal justificativa para isto seja a dificuldade de operacionalização deste tema nas pesquisas 3,5.

Serviços de saúde bucal foram, historicamente, de forma prioritária ofertados a escolares e frequentemente voltavam suas atenções para o público mais jovem; desta forma, a condição de saúde bucal encontrada em idosos no Brasil poderia ser mais satisfatória. Embora esse grupo seja beneficiado com algumas políticas públicas planejadas para toda a população, como a fluoretação da água, ainda existe pouca oferta de programas específicos para tais grupos 6 . A carência de políticas públicas em relação à população idosa, além da dificuldade de acesso odontológico para faixas etárias, aumenta seu grau de importância tendo em vista o rápido envelhecimento populacional observado no Brasil nas últimas décadas ${ }^{7}$, além da condição odontológica insatisfatória desta população ${ }^{8}$.

Segundo a Organização Mundial da Saúde (OMS), a situação de saúde bucal dos idosos é precária em muitos lugares do mundo, principalmente devido à falta de cuidado odontológico adequado, negligenciado por políticas públicas específicas 9 . O Brasil tem tentado, com relativo sucesso, enfrentar o problema, inclusive realizando levantamentos epidemiológicos em nível nacional, que quantificam as necessidades de saúde bucal e a capacidade de resposta dos serviços 10,11. Tais levantamentos indicaram importantes diferenças regionais em termos de utilização dos serviços de saúde bucal por idosos, como 22\% de uso no último ano para idosos na Região Norte e 31\% na Região Sudeste. Além disso, idosos que nunca consultaram esses serviços somam 28,5\% no Norte e 9,1\% no Sul do Brasil 12. Somado a isso, existe uma convergência dos resultados de algumas pesquisas que evidenciam menor utilização por populações mais pobres. A maioria dos estudos também mostra que a utilização predomina entre idosos mais escolarizados, que ainda possuem dentes e usuários de algum tipo de prótese 13,14,15.

Um dos esforços de maior destaque em termos de política pública para aumentar o acesso e a utilização de serviços de saúde bucal foi a inserção do dentista na equipe de saúde da família, por intermédio das equipes de saúde bucal. Tal política pretende a menor concentração dos profissionais nos grandes centros e o maior número de profissionais no SUS 16. Além disso, os Centros de Especialidades Odontológicas (CEOs) podem representar um atrativo a mais no serviço em função da resolução de necessidades de saúde mais complexas como próteses dentárias, lesões de mucosa bucal e doenças periodontais ${ }^{7}$. Considerando a escassez de informações sobre o acesso aos serviços de saúde no país e o envelhecimento populacional decorrente da transição demográfica e, em função da oferta de serviços tanto públicos quanto privados terem aumentado no Brasil, este trabalho objetiva medir a falta de acesso e a utilização dos serviços de saúde bucal por idosos da zona urbana do Município de Pelotas, Rio Grande do Sul.

\section{Métodos}

A população de Pelotas com mais de 60 anos, segundo o Censo Demográfico de 2010 (Instituto Brasileiro de Geografia e Estatística. http://www.ibge.gov.br), é composta por 60\% de mulheres, e 23\% dos idosos têm mais de 75 anos. Em relação à população total do município, 80\% se declaram com a cor da pele branca e $57 \%$ estão na classificação econômica C. 
Em termos de rede de saúde bucal, segundo dados da Sala de Apoio à Gestão Estratégica (Sage) do Ministério da Saúde, a cidade tem dois CEOs (Jequitibá e Sorrir), um laboratório regional de prótese dentária que até outubro de 2014 havia confeccionado 1.073 próteses para a população. Existem 52 equipes de Estratégia Saúde da Família (ESF) e 16 delas têm equipe de saúde bucal. Além disso, nos últimos dez anos houve um aumento do número de clínicas populares, que oferecem orçamento de baixo custo ou facilitado.

Este trabalho integra o consórcio de pesquisa 2013/2014 do Programa de Pós-graduação em Epidemiologia, Universidade Federal de Pelotas. A pesquisa utilizou delineamento transversal de base populacional. O processo de amostragem foi realizado em dois estágios. Inicialmente, foram selecionados os conglomerados (setores censitários) usando-se os dados do Censo Demográfico de 2010.

A seleção dos setores censitários foi sistemática, as probabilidades eram diferentes de acordo com o número de idosos e a renda média da família de cada setor. O maior tamanho de amostra estimada para este trabalho era de 1.089 idosos, mas, por se tratar de um consórcio de pesquisa e para contemplar o maior número necessário para os objetivos dos diferentes estudos, o número necessário foi de 1.649. Os domicílios particulares permanentes foram listados para a amostragem sistemática e dentre todos os setores foram selecionados 3.745 domicílios, aproximadamente 31 por setor, para alcançar uma amostra de 1.649 indivíduos na zona urbana do Município de Pelotas.

Todos os moradores com idade igual ou superior a 60 anos que não estavam hospitalizados, presos ou em instituições de longa permanência foram considerados elegíveis para o estudo. No caso de incapacidade de responder ao questionário, para os desfechos deste artigo, era permitida a resposta de um cuidador ou responsável. Foram excluídos do estudo aqueles que não tinham quem respondesse por eles.

As entrevistas foram feitas por pesquisadoras previamente treinadas com o auxílio de netbooks e verificação semanal de inconsistências com o supervisor de campo. Além disso, foi realizado controle de qualidade com $10 \%$ dos idosos por meio da aplicação de um questionário reduzido. A coleta de dados foi feita de janeiro a agosto de 2014.

Conforme mencionado brevemente na Introdução, os conceitos de utilização e acesso se confundem. Neste trabalho, buscou-se operacionalizar a medida de acesso como o indivíduo que buscou o serviço e não conseguiu utilizar no último ano, desta forma, é possível mensurar possíveis dificuldades que justifiquem a não utilização mesmo com a busca por serviço.

Utilizaram-se três variáveis dependentes: falta de utilização durante toda vida (sim/não), utilização odontológica no último ano (sim/não) e falta de acesso no último ano (sim/não). Ao abordar a questão da utilização dos serviços de saúde bucal, considerou-se duas dimensões: ao longo de toda vida e no último ano. Para a falta de acesso foi considerado apenas o último ano.

As variáveis de desfecho foram obtidas pelas seguintes perguntas: (i) falta de acesso no último ano: "O(a) $\mathrm{Sr}(\mathrm{a})$ buscou atendimento com dentista no último ano?". Se buscou, "O(a) $\mathrm{Sr}(\mathrm{a})$ conseguiu ser atendido?". A resposta negativa caracterizou falta de acesso; (ii) falta de utilização durante toda vida: "Alguma vez na vida o(a) Sr.(a) consultou com um dentista?"; e (iii) utilização no último ano: "Há quanto tempo foi a sua última consulta com dentista?”. As respostas foram categorizadas em menos de 1 ano e mais de 1 ano.

As variáveis de exposição foram: sexo (masculino; feminino), idade (60-64; 65-69; 70-74; 75 anos ou mais), escolaridade (Superior completo, 2 o grau completo ou Superior incompleto, 1o grau completo ou $2 \varrho$ incompleto, 1 ograu incompleto, 1a até $3 a$ série, nenhum), classificação econômica coletada segundo critérios da Associação Brasileira de Empresas de Pesquisas (ABEP. http://www.abep.org) e divididas em A/B; C; D/E, cor da pele (branca; amarela/preta/indígena/parda), situação conjugal (com companheiro; sem companheiro), trabalhando atualmente (sim; não), financiamento da última consulta (público; privado; convênio), marcação da consulta (telefone; pessoalmente ou ficha), problema na boca ou nos dentes (sim; não), necessidade de prótese (sim; não) e edentulismo (sim; não). Todas essas variáveis foram autorreferidas.

Para a análise ajustada, considerou-se um modelo hierárquico no qual as variáveis se dividiam em dois níveis: distal (sexo, idade, escolaridade, classificação econômica, cor da pele, situação conjugal, trabalhando atualmente) e proximal (problema nos dentes, precisa de prótese e edentulismo). A análise foi orientada por um modelo teórico, adaptado do modelo teórico de Andersen 5 que define as relações entre as variáveis sociodemográficas, odontológicas e do serviço em relação à utilização de serviços, valorizando a determinação social sobre o acesso e a utilização de serviços 14 . 
O controle de fatores de confusão foi realizado para as variáveis do mesmo nível e para as de níveis anteriores, sendo mantidas no modelo todas as variáveis. Para a análise estatística usou-se a regressão de Poisson, com o comando svy para levar em conta a amostragem em conglomerados do estudo. Inicialmente, foi realizada a análise bruta e em seguida a análise ajustada, ambas com intervalos de 95\% de confiança (IC95\%). Os valores de $\mathrm{p} \leq 0,05$ foram considerados estatisticamente significantes. O programa Stata versão 12.0 (https://www.stata.com) foi usado para a análise dos dados.

O presente trabalho foi realizado com o apoio da Coordenação de Aperfeiçoamento de Pessoal de Nível Superior (Capes; Código de Financiamento 001) e foi aprovado no Comitê de Ética da Faculdade de Medicina, Universidade Federal de Pelotas (protocolo no 201324538513.1.0000.5317, de 2013). Foi utilizado o Termo de Consentimento Livre Esclarecido atendendo-se a todas as peculiaridades éticas do estudo.

\section{Resultados}

Dos 1.844 elegíveis, foram entrevistados 1.451 idosos (21,3\% de perdas e recusas). A amostra foi composta predominantemente por indivíduos do sexo feminino (63\%), com 75 anos ou mais (29\%), da classe econômica C (52,5\%), cor da pele branca (83,7\%), com companheiro (50,5\%), que não estavam trabalhando $(80,4 \%)$, sem problemas nos dentes no último ano $(79,2 \%)$, que não necessitavam de prótese $(58,8 \%)$ e não edêntulos $(60,9 \%)$. Em relação à utilização de serviço odontológico, daqueles idosos que buscaram atendimento no último ano 98,2\% conseguiram ser atendidos, logo, a falta de acesso foi de $1,8 \%$. No que se refere à consulta alguma vez na vida foi verificado que 3,1\% dos idosos nunca tinham consultado com dentista, conforme Tabela 1 .

$\mathrm{Na}$ Tabela 2 estão incluídas as variáveis que descrevem a utilização no último ano. A marcação de consulta pelo telefone (67,3\%), o financiamento privado da última consulta $(67,8 \%)$, consultório particular (84,2\%) e o motivo rotina/manutenção (35,6\%) foram as características mais frequentes.

A Tabela 3 apresenta os resultados das análises bruta e ajustada considerando a utilização de serviço odontológico no último ano como variável dependente. As variáveis idade, escolaridade, situação conjugal, problema nos dentes no último ano, precisar de prótese e a ausência de dentes estiveram associadas com a utilização de serviços odontológicos tanto na análise bruta quanto na ajustada. $\mathrm{Na}$ análise ajustada, idosos com idades entre 65-70 anos apresentaram uma prevalência de utilização 44\% maior quando comparados com idosos com 75 anos ou mais. Em relação à escolaridade, idosos com ensino superior completo apresentaram uma prevalência de utilização 2,86 vezes maior quando comparados com idosos sem escolaridade. Já os idosos com companheiro(a) registraram uma prevalência de utilização $28 \%$ maior do que os(as) sem companheiro(a).

Idosos que relataram ter problemas nos dentes apresentaram uma prevalência de utilização 93\% maior do que aqueles sem problemas, já os indivíduos que precisavam de prótese apresentaram uma prevalência de utilização 36\% maior quando comparados com idosos que não precisavam. Constatouse também que idosos não edêntulos registraram uma prevalência de utilização 3,11 vezes maior do que os edêntulos.

A Figura 1 descreve a utilização odontológica e financiamento da última consulta no último ano em relação à classificação econômica e condição de edentulismo. O financiamento privado da utilização no último ano é maior do que em outros financiamentos e entre os edêntulos, exceto nas classes A/B nas quais estes grupos são semelhantes.

\section{Discussão}

A utilização de serviços odontológicos por idosos no último ano foi de 37\%, relativamente maior do que a de estudos de base populacional realizados no país nos últimos anos 15,17,18. Ainda assim, observou-se que $2 \%$ dos idosos que buscaram atendimento no último ano não obtiveram acesso aos serviços de saúde bucal. Em relação aos idosos que nunca consultaram com dentista na vida, o índice foi de aproximadamente 3\%, prevalência mais baixa do que a encontrada na literatura 19,20. 


\section{Tabela 1}

Descrição das características demográficas, socioeconômicas, odontológicas e financiamento. Pelotas, Rio Grande do Sul, Brasil, 2014 ( $N$ = 1.451).

\begin{tabular}{|c|c|c|}
\hline Variáveis & n & $\%$ \\
\hline \multicolumn{3}{|l|}{ Sexo } \\
\hline Feminino & 914 & 63,0 \\
\hline Masculino & 537 & 37,0 \\
\hline \multicolumn{3}{|l|}{ Idade (anos) } \\
\hline $60-64$ & 394 & 27,3 \\
\hline $65-69$ & 362 & 25,0 \\
\hline $70-74$ & 270 & 18,7 \\
\hline 75 ou mais & 420 & 29,0 \\
\hline \multicolumn{3}{|l|}{ Escolaridade } \\
\hline Superior completo & 157 & 10,9 \\
\hline 2o completo ou Superior incompleto & 159 & 11,1 \\
\hline 1 o completo ou 2 ㅇ incompleto & 143 & 9,9 \\
\hline 1 ㅇ grau incompleto & 445 & 31,1 \\
\hline 1ạ até 3ạ série & 337 & 23,4 \\
\hline Nenhuma & 196 & 13,6 \\
\hline \multicolumn{3}{|l|}{ Classificação econômica * } \\
\hline $\mathrm{A} / \mathrm{B}$ & 483 & 35,2 \\
\hline C & 720 & 52,5 \\
\hline $\mathrm{D} / \mathrm{E}$ & 169 & 12,3 \\
\hline \multicolumn{3}{|l|}{ Cor da pele } \\
\hline Branca & 1.211 & 83,7 \\
\hline Amarela/Preta/Indígena/Parda & 236 & 16,3 \\
\hline \multicolumn{3}{|l|}{ Situação conjugal } \\
\hline Com companheiro & 763 & 52,7 \\
\hline Sem companheiro & 684 & 47,3 \\
\hline \multicolumn{3}{|l|}{ Trabalhando atualmente } \\
\hline Não & 1.084 & 80,4 \\
\hline Sim & 264 & 19,6 \\
\hline \multicolumn{3}{|l|}{ Problema nos dentes } \\
\hline Não & 1.145 & 79,2 \\
\hline $\operatorname{Sim}$ & 300 & 20,8 \\
\hline \multicolumn{3}{|l|}{ Precisa de prótese } \\
\hline Não & 797 & 58,9 \\
\hline Sim & 556 & 41,1 \\
\hline \multicolumn{3}{|l|}{ Edêntulo } \\
\hline Não & 883 & 60,9 \\
\hline Sim & 566 & 39,1 \\
\hline \multicolumn{3}{|l|}{ Falta de utilização (toda a vida) } \\
\hline Não & 1.401 & 96,9 \\
\hline Sim & 45 & 3,1 \\
\hline \multicolumn{3}{|l|}{ Tempo da última consulta } \\
\hline Menos de 1 ano & 524 & 38,3 \\
\hline Mais de 1 ano & 843 & 61,7 \\
\hline \multicolumn{3}{|l|}{ Falta de acesso (último ano) } \\
\hline Não & 494 & 98,2 \\
\hline Sim & 9 & 1,8 \\
\hline
\end{tabular}

* Classificação econômica coletada segundo critérios da Associação Brasileira de Empresas de Pesquisas (ABEP.

http://www.abep.org). 


\section{Tabela 2}

Frequência das características estudadas nos idosos que utilizaram serviços de saúde bucal. Pelotas, Rio Grande do Sul, Brasil, $2014(n=524)$.

\begin{tabular}{lcc}
\hline Variáveis & $\mathbf{n}$ & $\%$ \\
\hline Como marcou a última consulta & & \\
$\quad$ Telefone & 346 & 67,3 \\
$\quad$ Pessoalmente ou por ficha & 168 & 32,7 \\
Financiamento da última consulta & 84 & 16,1 \\
$\quad$ Público & 352 & 67,8 \\
Privado & 84 & 16,1 \\
Convênio & & \\
Local da última consulta & 50 & 9,5 \\
Posto de Saúde & 20 & 3,9 \\
Centro de Especialidades Odontológicas & 6 & 1,2 \\
Faculdade de Odontologia & 438 & 84,2 \\
Consultório particular & 6 & 1,2 \\
Outros serviços & & \\
Motivo da última consulta & 184 & 35,6 \\
Rotina/Manutenção & 51 & 9,9 \\
Estava com dor & 92 & 17,8 \\
Ferida, caroço ou mancha na boca & 45 & 8,7 \\
Fazer dentadura & 152 & 28,0 \\
Outros motivos & & \\
\hline
\end{tabular}

Vale ressaltar uma limitação do estudo, que falta de acesso reflete apenas os indivíduos que buscaram efetivamente o serviço no último ano. Dessa forma, para a análise desses desfechos quase $60 \%$ da amostra foram desconsideradas, ainda que as necessidades de tratamento tenham sido levantadas no artigo para a amostra como um todo. Dos três desfechos analisados no estudo, apenas com a utilização no último ano foi feita análise multivariada. Esse fato se deve à baixa frequência de falta de acesso e de falta de utilização ao longo da vida.

Quando se refere à falta de acesso, isto pode ser atribuído principalmente à baixa busca de serviços de saúde bucal por parte dessa população. Em um estudo de metodologia semelhante e realizado na mesma cidade, os autores encontraram uma falta de acesso no último mês de 6,5\% para os serviços de saúde 21 , apesar do recordatório diferente e de avaliar outros serviços além da saúde bucal, e ao avaliar adultos e idosos fica evidente a menor procura por serviços de saúde bucal por parte da população, resultando em menos falta de acesso.

Existem poucos artigos que abordam a "falta de acesso" a serviços de saúde bucal em estudos de base populacional com idosos. Informações de uma pesquisa nacional mostraram uma falta de acesso odontológico no último ano de 11\% para indivíduos adultos na cidade de Porto Alegre, Rio Grande do Sul 22. A menor prevalência em idosos pode ser devido a uma menor percepção da necessidade de tratamento odontológico, principalmente entre os mais velhos e edêntulos. Além disso, a falta de acesso dos idosos pode ser menor considerando-se a prioridade em obter atendimento em função da legislação 23, a maior busca por serviços de saúde 24 , ou até mesmo a facilidade de fluxo no sistema de saúde decorrente do atendimento de outros problemas, como por exemplo, hipertensão arterial sistêmica e diabetes. No presente estudo, ao extrapolar para números absolutos, aproximadamente 16 mil idosos teriam buscado o dentista no último ano e, destes, 320 não conseguiram ser atendidos. Esse número mostra a relevância do esforço realizado na cidade em prol de aumento da rede de serviços de saúde bucal, particularmente no SUS e na ESF, além de refletir a importância das clínicas populares privadas no acesso a serviços de saúde bucal. 
Tabela 3

Razões de prevalência da utilização no último ano, de acordo com a análise bruta ou ajustada. Pelotas, Rio Grande do Sul, Brasil, 2014 ( $n$ = 1.367).

\begin{tabular}{|c|c|c|c|}
\hline Variável & $\begin{array}{c}\text { Utilização no último ano } \\
\text { n (\%) }\end{array}$ & RP bruta * (IC95\%) & RP ajustada ** (IC95\%) \\
\hline Sexo $* \star *$ & & $p=0,639$ & $p=0,292$ \\
\hline Masculino & $192(36,6)$ & 1,00 & 1,00 \\
\hline Feminino & $332(63,4)$ & $0,97(0,86-1,09)$ & $1,07(0,94-1,22)$ \\
\hline Idade (anos) $* \star *$ & & $\mathrm{p}<0,001 \#$ & $p=0,011 \#$ \\
\hline 75 ou mais & $104(20,0)$ & 1,00 & 1,00 \\
\hline $70-74$ & $99(19,0)$ & $1,41(1,13-1,76)$ & $1,20(0,96-1,50)$ \\
\hline $65-69$ & $154(29,6)$ & $1,71(1,37-2,12)$ & $1,44(1,15-1,79)$ \\
\hline $60-64$ & $164(31,5)$ & $1,59(1,28-1,98)$ & $1,22(0,96-1,55)$ \\
\hline Escolaridade $* * *$ & & $\mathrm{p}<0,001 \#$ & $p=0,001 \#$ \\
\hline Nenhuma & $36(7,0)$ & 1,00 & 1,00 \\
\hline 1ạ até 3ạ série & $85(16,4)$ & $1,28(0,91-1,81)$ & $1,25(0,88-1,79)$ \\
\hline 1ㅇg grau incompleto & $128(24,8)$ & $1,47(1,06-2,05)$ & $1,22(0,85-1,76)$ \\
\hline 1 o completo ou 2 o incompleto & $59(11,4)$ & $2,01(1,43-2,84)$ & $1,70(1,16-2,50)$ \\
\hline 2o completo ou Superior incompleto & $89(17,2)$ & $2,71(1,94-3,78)$ & $2,02(1,38-2,96)$ \\
\hline Superior completo & $120(23,2)$ & $3,72(2,73-5,07)$ & $2,86(1,96-4,17)$ \\
\hline Classificação econômica *** & & $\mathrm{p}<0,001 \#$ & $p=0,055 \#$ \\
\hline $\mathrm{D} / \mathrm{E}$ & $34(6,9)$ & 1,00 & 1,00 \\
\hline $\mathrm{C}$ & $204(41,6)$ & $1,31(0,92-1,84)$ & $1,02(0,72-1,46)$ \\
\hline $\mathrm{A} / \mathrm{B}$ & $253(51,5)$ & $2,37(1,73-3,24)$ & $1,30(0,90-1,85)$ \\
\hline Cor da pele ${ }^{* * *, \# \#}$ & & $p=0,980$ & $p=0,262$ \\
\hline Amarela/Preta/Indígena/Parda & $84(16,1)$ & 1,00 & 1,00 \\
\hline Branca & $438(83,9)$ & $0,99(0,81-1,23)$ & $0,89(0,73-1,09)$ \\
\hline Situação conjugal *** & & $p=0,030$ & $p=0,002$ \\
\hline Sem companheiro & $207(39,7)$ & 1,00 & 1,00 \\
\hline Com companheiro & $315(60,3)$ & $1,34(1,02-1,58)$ & $1,28(1,09-1,49)$ \\
\hline Trabalhando atualmente $* * \star$ & & $p=0,007$ & $p=0,605$ \\
\hline Não & $375(75,9)$ & 1,00 & 1,00 \\
\hline Sim & $119(24,1)$ & $1,25(1,06-1,47)$ & $0,96(0,81-1,14)$ \\
\hline Problema nos dentes último ano \#\#\# & & $p<0,001$ & $p<0,001$ \\
\hline Não & $197(37,7)$ & 1,00 & 1,00 \\
\hline Sim & $326(62,3)$ & $2,21(1,94-2,52)$ & $1,93(1,66-2,23)$ \\
\hline Precisa de prótese \#\#\# & & $p=0,009$ & $p<0,001$ \\
\hline Não & $294(64,3)$ & 1,00 & 1,00 \\
\hline Sim & $163(35,7)$ & $1,26(1,06-1,50)$ & $1,36(1,15-1,60)$ \\
\hline Edêntulo \#\#\# & & $p<0,001$ & $p<0,001$ \\
\hline Sim & $73(13,9)$ & 1,00 & 1,00 \\
\hline Não & $451(86,1)$ & $3,85(3,01-4,93)$ & $3,11(2,37-4,09)$ \\
\hline
\end{tabular}

* Análise realizada com regressão de Poisson e variância corrigida para desenho do estudo;

** Análise ajustada para mesmo nível e superior;

*** Nível distal;

\# Valor de p de tendência linear;

\#\# Classificação econômica coletada segundo critérios da Associação Brasileira de Empresas de Pesquisas (ABEP. http://www.abep.org);

\#\#\# Nível proximal. 
Figura 1

Utilização odontológica no último ano de acordo com a classificação econômica *, edentulismo e financiamento da consulta. Pelotas, Rio Grande do Sul, Brasil.

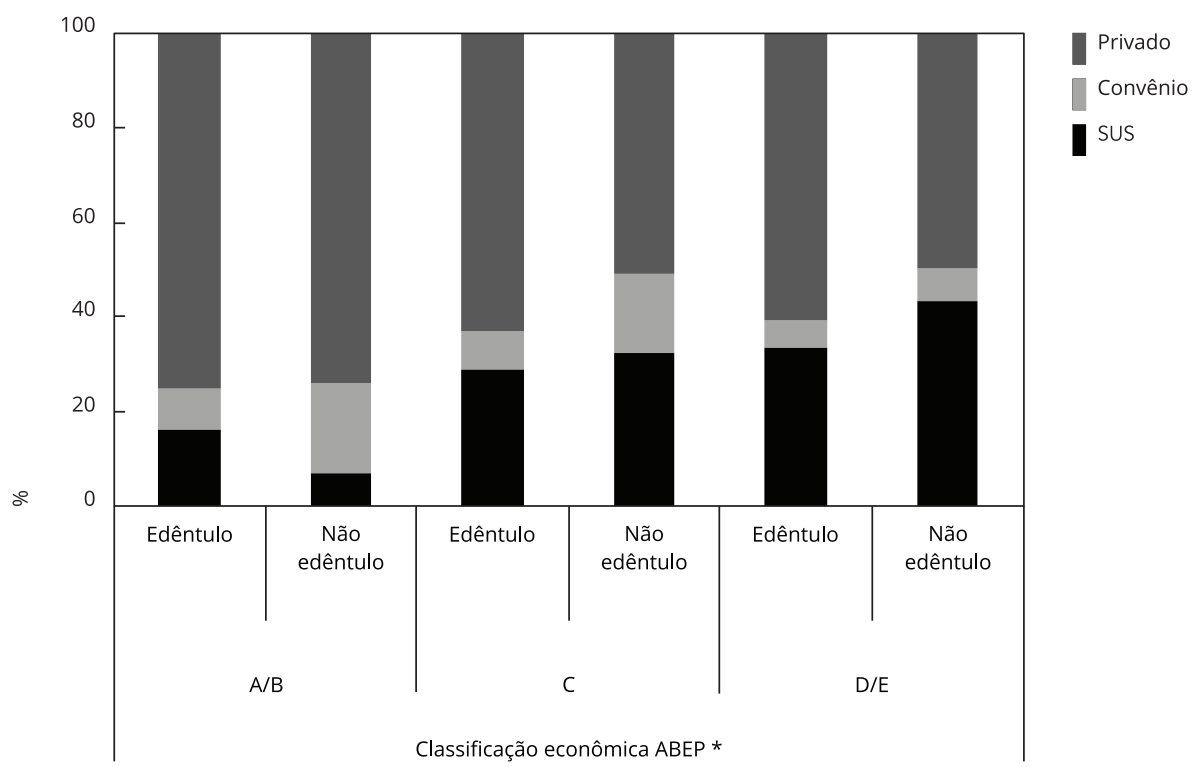

* Classificação econômica coletada segundo critérios da Associação Brasileira de Empresas de Pesquisas (ABEP. http://www.abep.org).

O desfecho "idosos que nunca consultaram na vida" é um proxy de falta de acesso, seja por fatores individuais, por fatores sociais ou do serviço. No Brasil, as prevalências encontradas pela maioria dos artigos são inferiores a 10\%, sendo 5,8\% 25 em um estudo de abrangência nacional e 6,3\% 6 em estudo no Estado de São Paulo. Essa medida ainda que pequena em nosso meio é preocupante, pois dimensiona a falta de acesso histórica a serviços odontológicos da população brasileira, já salientada em outros artigos 22,26. Em números absolutos, considerando-se os dados do censo 2010, aproximadamente 500 idosos na cidade de Pelotas nunca foram ao dentista.

O uso de serviços odontológicos foi observado somente em relação ao tempo (menos de 1 ano; mais de 1 ano), não discriminando o tipo de necessidade, se problema eletivo, preventivo ou de urgência. A periodicidade das consultas é uma controvérsia na literatura. Alguns estudos categorizam o tempo de uso de formas diferentes (menos de 1 ano 16,19; menos de 2 anos 13; menos de 3 anos 15) com a intenção de estabelecer parâmetros de referência para a ida regular ao dentista, considerando suas contribuições às condições de saúde bucal e geral dos indivíduos. Neste estudo, optou-se pelo pressuposto de que todos os idosos deveriam ir ao dentista ao menos uma vez por ano, período adequado aos objetivos de prevenção e cuidados de saúde bucal, além de seus reflexos na saúde geral 27 .

Observou-se que idosos com maior grau de escolaridade apresentaram maior utilização dos serviços odontológicos no último ano, achado corroborado por outras evidências nacionais 18,25. A escolaridade promove maior acesso à informação e melhor percepção da saúde, além de ser um marcador de condição econômica, oportunizando uma maior busca e utilização dos serviços de saúde, tanto médicos quanto odontológicos 15. Já para a idade e situação conjugal dos idosos, em acordo com outros artigos, identificou-se uma maior utilização no último ano em idosos com menores idades e que possuíam companheiro em relação aos de maiores idades e que não possuíam companheiro 12,13.

A maior utilização por idosos de menor idade pode ser decorrente de uma maior necessidade de saúde, devido à maior presença de dentes neste grupo (dados não apresentados). Além disso, o modelo 
assistencial odontológico mudou sua filosofia nas últimas décadas para um viés mais preventivo. Os idosos mais jovens podem ter recebido mais cuidados preventivos em relação à saúde bucal em diferentes períodos históricos 25,28 . Idosos com companheiro podem usar mais os serviços de saúde bucal em função tanto do apoio como do estímulo exercido pelo companheiro na percepção e no cuidado à saúde 29,30 .

Idosos com problema bucal ou com necessidade de prótese consultaram mais no último ano do que idosos sem estas condições, à semelhança de outros estudos 18,29,30. Esse resultado pode ser em parte explicado pelo aumento da oferta de próteses dentárias no município a partir de 2012. O edentulismo é considerado o principal preditor para a falta de utilização odontológica regular 6,25,28. Neste estudo, observou-se um elevado edentulismo (40\%), que mostrou a maior medida de efeito nas análises bruta e ajustada para a não utilização de serviço de saúde bucal.

A classificação econômica perdeu significância estatística em relação ao uso no último ano quando controlada para outras variáveis socioeconômicas e demográficas, contradizendo a predominância da literatura 13,14,19. O aumento da oferta de serviços odontológicos públicos e privados, concomitantemente com o aumento da renda média da população, parece ser a explicação mais plausível para esse achado, promovendo a equidade na utilização de serviços odontológicos em Pelotas. Em números absolutos, utilizando os dados do censo 2010, 16 mil idosos teriam consultado com dentista no último ano, sendo aproximadamente 10 mil no serviço particular, 3 mil por convênio e 3 mil pelo serviço público. Esses dados mostram que ainda que a rede de serviços da cidade tenha sido expandida, um contingente importante de indivíduos ainda usa o setor privado como forma de acessar os serviços de saúde bucal.

Algumas limitações do estudo devem ser destacadas. O período recordatório de um ano para a avaliação do acesso aos serviços de saúde bucal, frequente na literatura 16,19, pode subestimar as prevalências, embora não se disponha de indicativo de distorção das medidas de efeito 31 . O viés de seleção pode estar presente em função do percentual de perdas e recusas. Entretanto, não ocorreu uma aglomeração de perdas e recusas em alguma região da cidade de Pelotas ou na faixa etária da amostra estudada. Além disso, as prevalências encontradas para as variáveis sociodemográficas em Pelotas pelo último censo estão próximas dos valores encontrados na população investigada neste trabalho.

Dentre as contribuições do artigo salienta-se a avaliação do acesso a serviços de saúde bucal por idosos, em um estudo de base populacional, fornecendo estimativas relevantes para os gestores de um grupo etário ainda pouco investigado, apesar de sua crescente importância social e demográfica.

Neste trabalho, a falta de acesso e a falta de utilização ao longo da vida foram pouco prevalentes, entretanto, o uso dos serviços de saúde bucal no último ano foi mais alto do que o registrado em outros artigos da literatura. A expansão da rede de serviços públicos e o acesso a serviços privados de baixo custo parecem explicar esse achado. Identifica-se a necessidade de contínua ampliação de investimentos públicos no sentido de incrementar o acesso de idosos a serviços odontológicos pelo SUS, particularmente com o maior fornecimento de próteses dentárias, melhorando, assim, a qualidade de vida dos indivíduos desdentados de modo equitativo. 


\section{Colaboradores}

G. S. Sória, B. P. Nunes e L. A. Facchini participaram da concepção, planejamento, análise, interpretação, redação e aprovação final do trabalho. C. S. Bavaresco e L. S. Vieira colaboraram na redação, revisão e aprovação final do trabalho.

\section{Informações adicionais}

ORCID: Giordano Santana Sória (0000-00016932-1265); Bruno Pereira Nunes (0000-00024496-4122); Caren Serra Bavaresco (0000-00020730-3632); Luna Strieder Vieira (0000-00019946-8575); Luiz Augusto Facchini (0000-00025746-5170)

\section{Agradecimentos}

Agradecemos à Coordenação de Aperfeiçoamento de Pessoal de Nível Superior (Capes) e à Fundação de Amparo à Pesquisa do Estado do Rio Grande do Sul (FAPERGS) pelas bolsas de mestrado.

\section{Referências}

1. Paim J, Travassos C, Almeida C, Bahia L, Macinko J. The Brazilian health system: history, advances, and challenges. Lancet 2011; 377:1778-97.

2. Oliver A, Mossialos E. Equity of access to health care: outlining the foundations for action. J Epidemiol Community Health 2004; 58:655-8.

3. Travassos C, Martins M. Uma revisão sobre os conceitos de acesso e utilização de serviços de saúde. Cad Saúde Pública 2004; 20 Suppl 2:S190-8.

4. Donabedian A. An introduction to quality assurance in health care. Oxford: Oxford University Press; 2002.

5. Andersen RM. National health surveys and the behavioral model of health services use. Med Care 2008; 46:647-53.

6. Gibilini C, Esmeriz CEC, Volpato LF, Meneghim ZMAP, Silva DD, Sousa MLR. Acesso a serviços odontológicos e auto-percepção da saúde bucal em adolescentes, adultos e idosos. Arq Odontol 2010; 46:213-23.

7. Oliveira RFR, Souza JGS, Haikal DS, Ferreira EF, Martins AMEBL. Equity inthe use of dental services provided by the Brazilian Unified Health System (SUS) among the elderly: a population-based study. Ciênc Saúde Colet 2016; 21:3509-23.

8. Braine T. More oral health care needed for ageing populations. Bull World Health Organ 2005; 83:646-7.

9. Oliveira TC, Silva DA, Freitas YNL, Silva RL, Pegado CP, Lima KC. Socio-demographic factors and oral health conditions in the elderly: a population-based study. Arch Gerontol Geriatr 2013; 57:389-97.

10. Azevedo JS, Azevedo MS, Oliveira LJC, Correa $\mathrm{MB}$, Demarco FF. Uso e necessidade de prótese dentária em idosos brasileiros segundo a Pesquisa Nacional de Saúde Bucal (SBBrasil 2010): prevalências e fatores associados. Cad Saúde Pública 2017; 33:e00054016.

11. Mendes DC, Poswar FDE, Oliveira MV, Haikal DS, Silveira MF, Martins AM, et al. Analysis of socio-demographic and systemic health factors and the normative conditions of oral health care in a population of the Brazilian elderly. Gerodontology 2012; 29:e206-14.

12. Departamento de Atenção Básica, Secretaria de Atenção à Saúde, Ministério da Saúde. Projeto SB-Brasil 2010. Brasília: Ministério da Saúde; 2011.

13. Ferreira CDO, Antunes JLF, Andrade FBD. Fatores associados à utilização dos serviços odontológicos por idosos brasileiros. Rev Saúde Pública 2013; 47:90-7.

14. Macinko J, Lima-Costa MF. Horizontal equity in health care utilization in Brazil, 1998-2008. Int J Equity Health 2012; 11:33.

15. Silva AE, Langlois CDE, Feldens CA. Use of dental services and associated factors among elderly in southern Brazil. Rev Bras Epidemiol 2013; 16:1005-16. 
16. Camargo MBJ, Dumith SC, Barros AJD. Uso regular de serviços odontológicos entre adultos: padrões de utilização e tipos de serviços. Cad Saúde Pública 2009; 25:1894-906.

17. Baldani MH, Antunes JLF. Inequalities in access and utilization of dental services: a crosssectional study in an area covered by the Family Health Strategy. Cad Saúde Pública 2011; 27 Suppl 2:S272-83.

18. Roncalli AG. The SB Brasil 2010 Project: a key strategy for developing an oral health surveillance model. Cad Saúde Pública 2010; 26:428-9.

19. Araújo CS, Lima RC, Peres MA, Barros AJD. Utilização de serviços odontológicos e fatores associados: um estudo de base populacional no Sul do Brasil. Cad Saúde Pública 2009; 25:1063-72.

20. Manhães ALD, Costa AJL. Acesso a e utilização de serviços odontológicos no Estado do Rio de Janeiro, Brasil, em 1998: um estudo exploratório a partir da Pesquisa Nacional por Amostra de Domicílios. Cad Saúde Pública 2008; 24:207-18.

21. Nunes BP, Thumé E, Tomasi E, Duro SMS, Facchini LA. Desigualdades socioeconômicas no acesso e qualidade da atenção nos serviços de saúde. Rev Saúde Pública 2014; 48:968-76.

22. Peres MA, Iser BPM, Boing AF, Yokota RTC, Malta DC, Peres KG. Desigualdades no acesso e na utilização de serviços odontológicos no Brasil: análise do Sistema de Vigilância de Fatores de Risco e Proteção para Doenças Crônicas por Inquérito Telefônico (VIGITEL 2009). Cad Saúde Pública 2012; 28 Suppl:S90-100.

23. Brasil. Lei no 10.741, de 1o de outubro de 2003. Dispõe sobre o Estatuto do Idoso e dá outras providências. Diário Oficial da União 2003; 3 out.
24. Pinheiro RS, Viacava F, Travassos C, Brito ADS. Gênero, morbidade, acesso e utilização de serviços de saúde no Brasil. Ciênc Saúde Colet 2002; 7:687-707.

25. Martins AMEDBL, Barreto SM, Pordeus IA. Uso de serviços odontológicos entre idosos brasileiros. Rev Panam Salud Pública 2007; 22:308-16.

26. Monteiro CN, Beenackers MA, Goldbaum M, Azevedo Barros MB, Gianini RJ, Cesar $\mathrm{CL}$, et al. Socioeconomic inequalities in dental health services in São Paulo, Brazil, 20032008. BMC Health Serv Res 2016; 16:683.

27. Gilbert GH, Stoller EP, Duncan RP, Earls JL, Campbell AM. Dental self-care among dentate adults: contrasting problem-oriented dental attenders and regular dental attenders. Spec Care Dentist 2000; 20:155-63.

28. Matos DL, Lima-Costa MF. Tendência na utilização de serviços odontológicos entre idosos brasileiros e fatores associados: um estudo baseado na Pesquisa Nacional por Amostra de Domicílios (1998 e 2003). Cad Saúde Pública 2007; 23:2740-8.

29. Holm-Pedersen P, Vigild M, Nitschke I, Berkey DB. Dental care for aging populations in Denmark, Sweden, Norway, United kingdom, and Germany. J Dent Educ 2005; 69:987-97.

30. Listl S. Income-related inequalities in dental service utilization by Europeans aged $50+$. J Dent Res 2011; 90:717-23.

31. Rothman KJ, Greenland S, Lash TL. Modern epidemiology. Philadelphia: Lippincott Williams \& Wilkins; 2008. 


\section{Abstract}

This article sought to measure lack of access and use of oral health services by elderly residents of Pelotas, Rio Grande do Sul State, Brazil. This is a cross-sectional population study carried out in 2014 , in the city's urban zone, including individuals aged 60 years or more. Sociodemographic and self-reported needs variables were associated with outcomes. We used Poisson regression for the crude and adjusted analyses. We interviewed 1,451 elderly individuals using a structured questionnaire. The prevalence of lack of access in the previous year was of 1.8\% (95\%CI: 0.7-3.0). Elderly individuals who never had a consultation were 3.1\% (95\% CI: 2.2-4.0) and the prevalence of use of oral health services in the previous years was 38.3\%(95\%CI 36.0-41.0). Use in the previous year was positively associated with the following variables: younger age $(P R=1.16)$, having a partner $(P R=1.28)$, high educational level $(P R=$ $1.31)$, mouth or teeth problems $(P R=1.93)$, need for dental prosthesis $(P R=1.36)$ and being edentulous $(P R=3.11)$. Lack of access in the previous year was low. Oral health service use was higher than that observed in other states. Findings seem to reflect the expansion of oral health services in the city, especially in the public network, and are useful for health policy planning.

Health Services Accessibility; Equity in Access to Health Services; Health Services for the Aged; Dental Health Services

\section{Resumen}

El objetivo fue medir la falta de acceso y utilización de los servicios de salud bucal por parte de ancianos en Pelotas, Río Grande do Sul, Brasil. Se realizó un estudio transversal de base poblacional durante el año 2014, en la zona urbana del municipio, incluyendo a individuos con 60 años y más. Las variables sociodemográficas y de necesidad autorreferidas se asociaron a los resultados. Se utilizó la regresión de Poisson para los análisis bruto y ajustado. Se entrevistaron a 1.451 ancianos, mediante un cuestionario estructurado. La falta de acceso en el último año alcanzó una prevalencia de 1,8\% (IC95\%: 0,7-3,0). Los ancianos que nunca consultaron sumaron un 3, 1\% (IC95\%: 2,2-4,0) y la utilización de servicios de salud bucal en el último año tuvo una prevalencia de un 38,3\% (IC95\%: 36,0-41,0). La utilización durante el último año presentó una asociación positiva con las siguientes variables: franja de edad más joven $(R P=1,16)$, tener compañero $(R P=1,28)$, alta escolaridad $(R P=1,31)$, problema en la boca o en los dientes $(R P=1,93)$, necesidad de prótesis dental $(R P=1,36)$ y ser edéntulo $(R P=3,11)$. La falta de acceso en el último año fue baja. La utilización de servicios de salud bucal fue más alta que la observada en otros estudios. Los resultados parecen reflejar la expansión de los servicios de salud bucal en el municipio, particularmente en la red pública, siendo útiles para las acciones de planificación dentro de las politicas de salud.

Accesibilidad a los Servicios de Salud; Equidad en el Acceso a los Servicios de Salud; Servicios de Salud para Ancianos; Servicios de Salud Dental
Recebido em 03/Out/2018

Versão final reapresentada em 04/Jan/2019 Aprovado em 11/Jan/2019 